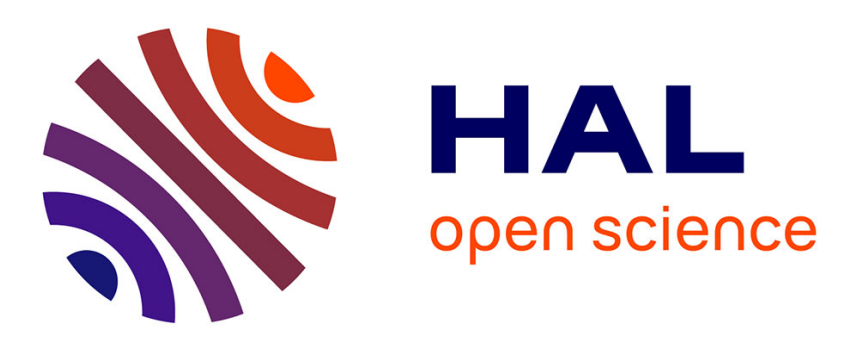

\title{
High resolution 13C NMR investigation in the organic conductor : (TMTSF)2ClO4
}

P. Bernier, M. Audenaert, R.J. Schweizer, P.C. Stein, D. Jérome, K. Bechgaard, A. Moradpour

\section{- To cite this version:}

P. Bernier, M. Audenaert, R.J. Schweizer, P.C. Stein, D. Jérome, et al.. High resolution 13C NMR investigation in the organic conductor: (TMTSF)2ClO4. Journal de Physique Lettres, 1985, 46 (15), pp.675-681. 10.1051/jphyslet:019850046015067500 . jpa-00232579

\section{HAL Id: jpa-00232579 https://hal.science/jpa-00232579}

Submitted on 1 Jan 1985

HAL is a multi-disciplinary open access archive for the deposit and dissemination of scientific research documents, whether they are published or not. The documents may come from teaching and research institutions in France or abroad, or from public or private research centers.
L'archive ouverte pluridisciplinaire HAL, est destinée au dépôt et à la diffusion de documents scientifiques de niveau recherche, publiés ou non, émanant des établissements d'enseignement et de recherche français ou étrangers, des laboratoires publics ou privés. 
Classification

Physics Abstracts

76.60C

\title{
High resolution ${ }^{13} \mathrm{C}$ NMR investigation in the organic conductor : $\left(\mathrm{TMTSF}_{2} \mathrm{ClO}_{4}\right.$
}

\author{
P. Bernier, M. Audenaert, R. J. Schweizer ${\left({ }^{*}\right)}^{*}$ \\ Groupe de Dynamique des Phases Condensées, USTL, 34060 Montpellier, France \\ P. C. Stein, D. Jérome, K. Bechgaard and A. Moradpour \\ Laboratoire de Physique des Solides, Université Paris Sud, 91405 Orsay Cedex, France
}

(Reçu le 3 avril 1985, accepté le 11 juin 1985)

\begin{abstract}
Résumé. - Nous présentons des résultats de $\mathrm{RMN} \mathrm{du}{ }^{13} \mathrm{C}$ obtenus avec rotation à l'angle magique sur la molécule TMTSF (en solution et à l'état solide) et dans le conducteur organique (TMTSF) ${ }_{2} \mathrm{ClO}_{4}$. Les données (déplacement de Knight, $T_{1}$ et $T_{1 \rho}$ ) permettent d'identifier tous les sites individuels de la molécule. L'observation d'une large distribution et d'une forte augmentation du taux de relaxation des spins des différents sites est en relation avec le rôle important joué par les interactions Coulombiennes, en particulier pour les noyaux situés aux quatre coins de la molécule.

Abstract. - We report the results of ${ }^{13} \mathrm{C}$ NMR magic angle spinning experiments performed on the TMTSF molecule (in solution and in the solid state) and in the organic conductor (TMTSF) ${ }_{2} \mathrm{ClO}_{4}$. This investigation (Knight-shift, $T_{1}$ and $T_{1 \rho}$ ) has allowed to resolve all individual sites of the organic molecule. Strong and widely distributed enhancements of the relaxation rate of the nuclear spin on different sites of the molecule suggest a picture where Coulomb correlations play a major role for the sites at the corners of the molecule.
\end{abstract}

\section{Introduction.}

The observation « at the microscopic level » of the properties of metals is of relevance if we want to describe details of their band structure and other electronic properties. In molecular conductors even more so, because their conduction band arises from diffuse (delocalized) molecular orbitals. In this Letter we report the first high-resolution ${ }^{13} \mathrm{C} N \mathrm{~N}$ investigation of solid (TMTSF) ${ }_{2} \mathrm{ClO}_{4}$ [1]. We have determined spin-lattice relaxation times, $T_{1}$, relaxation times in the rotating frame, $T_{1 \rho}$, and $\mathrm{Knight}$-shifts of well resolved ${ }^{13} \mathrm{C}$ resonance lines. We have assigned individual lines (or groups of lines) to the various carbon sites of the TMTSF molecule. The spectra reflect the low symmetry of the (TMTSF) ${ }_{2} \mathrm{ClO}_{4}$ structure. We have correlated the calculated properties for the molecular building block $\mathrm{TMTSF}^{+0.5}$, in particular spin-densities, with those observed in the conducting solid. Furthermore, we have found striking deviations from the classical Korringa-type behaviour.

$\left(^{*}\right)$ On leave from the Max-Planck-Institut für Festkörperforschung, Stuttgart, F.R.G. 
Recently, a similar analysis has been reported for the organic conductor di-(fluoranthenyl)ium-hexafluoroantimonate [2].

\section{Experimental.}

(TMTSF) ${ }_{2} \mathrm{ClO}_{4}$ [1] and TMTSF [3] were obtained by usual procedures. The NMR of powdered samples has been investigated in deuterated PMMA Andrew-like rotors. All the measurements were performed at room temperature using a Bruker CXP 200 spectrometer working at $f=$ $50.3 \mathrm{MHz}$. Magic angle spinning $\left(f_{\mathrm{R}}=3 \mathrm{kHz}\right)$, proton enhancement and proton decoupling were necessary to achieve good sensitivity and high resolution [4]. Typical ${ }^{13} \mathrm{C}$ linewidths obtained in our samples were in the range of 2 to $4 \mathrm{ppm}(0.1$ to $0.4 \mathrm{kHz})$.

The shift values reported below are measured with respect to the classical reference tetramethylsilane (TMS) [4].

For the $T_{1}$ relaxation measurements a negative longitudinal ${ }^{13} \mathrm{C}$ magnetization was first prepared using a standard proton enhancement sequence followed by a $\pi / 2$ pulse which was subsequently monitored at variable delay times. The observation of the central carbon atoms in solid (TMTSF) ${ }_{2} \mathrm{ClO}_{4}$ (1 and 3 in Fig. 1) has required a different approach. In the neutral molecule the contact with the protons is sufficiently efficient to give a significant ${ }^{13} \mathrm{C}$ polarization. In the conducting salt, however, these central carbons relax so rapidly that proton enhancement is no longer efficient, and thus a standard $\pi / 2$ pulse sequence has to be used. $T_{1 \rho}$ measurements needed ${ }^{13} \mathrm{C}$ spin-lock with variable delay in addition to the proton enhancement pulse sequence.

Parasitic signals have been carefully identified as coming from the rotor and the sample container. Usually these signals were broad, of small amplitude and restricted to the 0-190 ppm region of chemical shifts.

\section{Results and discussion.}

3.1 NeUTRAL TMTSF. - The spectra of neutral TMTSF in solution and in the solid state are shown in figure $2 a$ and $b$, respectively. The individual $j$-carbon atoms are labelled according to figure 1 . In solution we expect and observe three lines corresponding to centre-, corner- and
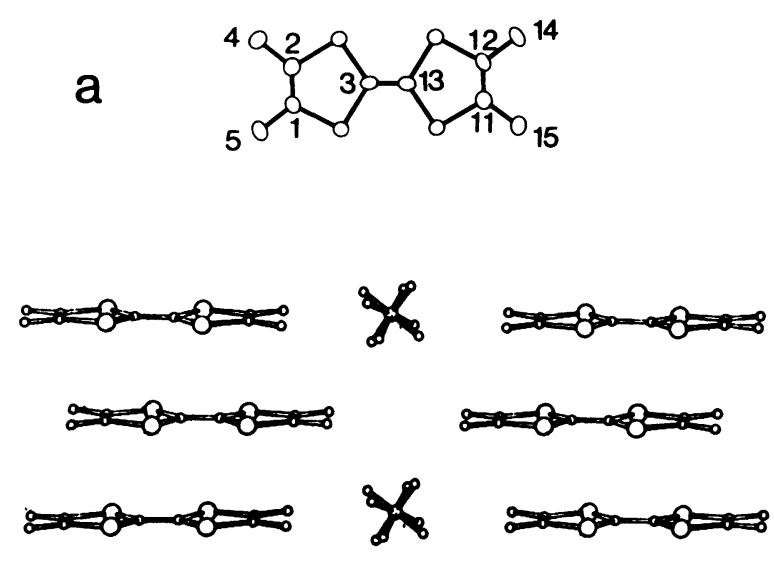

b

Fig. 1. - The TMTSF molecule with the carbon indexation (a). Stacking of the TMTSF molecules in the salt (TMTSF) ${ }_{2} \mathrm{ClO}_{4}(\mathrm{~b})$. 


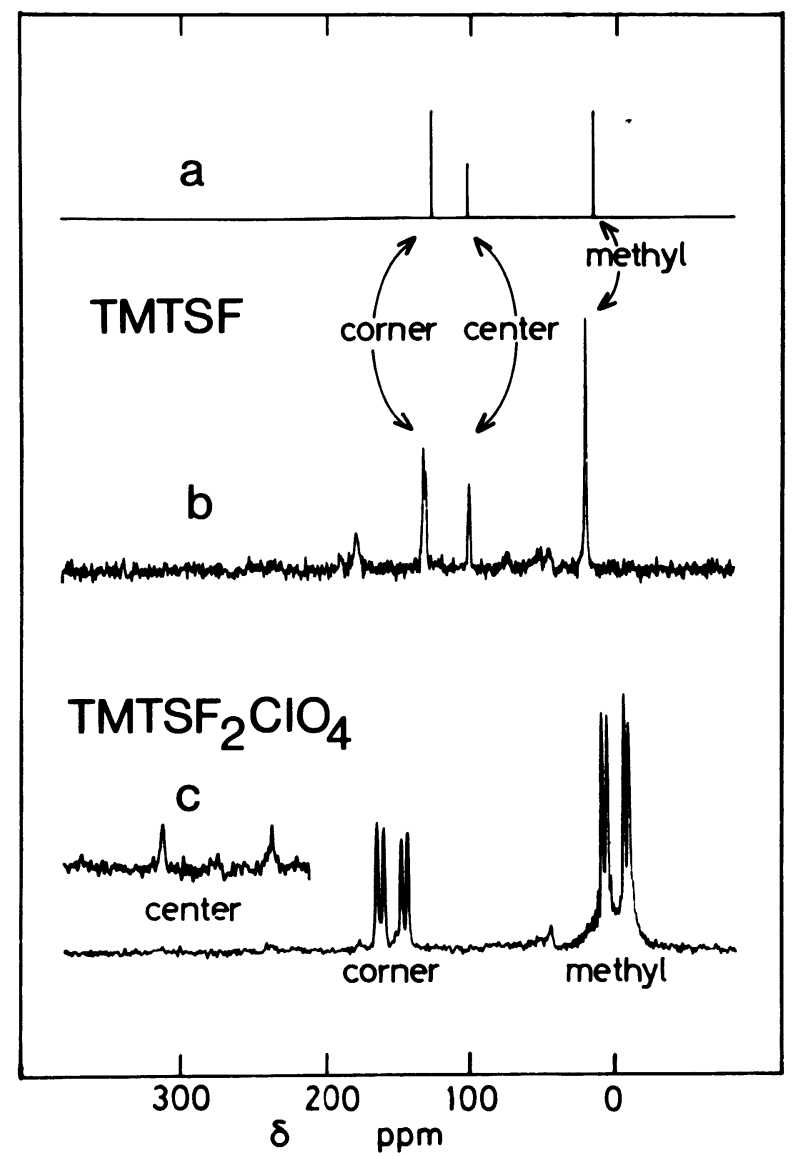

Fig. 2. $-{ }^{13} \mathrm{C}$ high resolution NMR spectrum of TMTSF in liquid $\mathrm{CHCl}_{3}$ solution (a), in the solid state (b) and in the conducting salt (c).

methyl-carbons with 2:4:4 intensity respectively. Solid TMTSF crystallizes in space group Pi [5] and for symmetry reasons we expect 5 lines : 1 centre, 2 corner and 2 methyl lines. We assign the nearly identical spectra in figure 2 as follows (see Table I) :

Table I. - Chemical shifts and $T_{1 \rho}$ values for the different carbon positions of the TMTSF molecule in the liquid and solid states. $T_{1}$ values $($ at $50.3 \mathrm{MHz})$ are larger than $60 \mathrm{~s}$.

\begin{tabular}{|c|c|c|c|c|}
\hline $\begin{array}{c}\text { Position } \\
\text { in TMTSF molecule }\end{array}$ & $\begin{array}{r}C \\
\text { liquid } \\
\text { (ppm) }\end{array}$ & $\begin{array}{l}\text { ift } \\
\text { solid } \\
\text { (ppm) }\end{array}$ & $\begin{array}{r}T_{1 \rho} \\
(\mathrm{ms})\end{array}$ & $\begin{array}{l}\text { relative } \\
\text { intensity }\end{array}$ \\
\hline $4,5,14,15$ & 15 & 18 & 8 & 4 \\
\hline 3,13 & 102 & 99 & 220 & 2 \\
\hline $1,2,11,12$ & 126 & 130 & 70 & 4 \\
\hline
\end{tabular}


(i) $T_{1 \rho j}$ is expected to increase when the distance between the $j$-carbon nucleus and the protons increases, as it arises from the proton-carbon dipolar coupling. ( $T_{1}$ for the neutral TMTSF molecule is very long for all $j$ 's and has been estimated $>60 \mathrm{~s}$.)

(ii) Relative intensities $(4: 2: 4)$ agree with the relative number of carbon atoms for methyl, centre and corner carbons of the molecule, respectively (see Table I).

(iii) We have assigned the line at $18.4 \mathrm{ppm}$ to the methyl carbons, using the standard gated decoupling technique [4].

We did not observe the expected splitting of the methyl carbons at $18 \mathrm{ppm}$, but there is some indication of a splitting in the $130 \mathrm{ppm}$ (corner) carbons. All in all, as solid state and solution spectra agree well, we feel justified to use the assigned shifts when calculating Knight-shifts (Table II).

3.2 (TMTSF) ${ }_{2} \mathrm{ClO}_{4}$. - Solid (TMTSF) ${ }_{2} \mathrm{ClO}_{4}$ crystallizes with the triclinic symmetry (space group $\mathrm{P} \overline{1})$ with the $\mathrm{ClO}_{4}^{-}$anions sitting at inversion centres [6]. The three-dimensional unit cell contains therefore two TMTSF molecules along the stacking axis. The molecules are dimerized, so to speak, along the stacking axis. This dimerization is often characterized by the difference between mean intermolecular distances [6]. There are 20 different carbon sites in the threedimensional unit-cell, but the crystalline symmetry reduces the number of independent sites to 10. The existence of Knight-shifts in the conducting salt should resolve the ten carbon resonances in three groups of 4, 4, and 2 lines, respectively. As demonstrated in figure 2 and table II, this is indeed the case. The group of 4 lines close to $0 \mathrm{ppm}$ is attributed to the methyl carbons as their rotating frame relaxation is faster (due to methyl rotation) than the relaxation of the two other groups. Consequently, the group of 4 equally intense lines around $150 \mathrm{ppm}$ is assigned to the corner C-atoms. Finally. the two resonance lines at 240 and $310 \mathrm{ppm}$ are assigned to the central carbons, which exhibit the largest shifts.

The shifts listed in table II can therefore be considered as a consequence of the conducting character of this molecular compound (Knight-shift).

In general, the magnitude of a Knight-shift, $K_{j}$, can be correlated to the local spin densities through the relation:

$$
K_{j}=\chi_{\mathrm{P}} \frac{A_{j} \rho_{j}}{\hbar \gamma_{\mathrm{e}} \gamma_{\mathrm{n}}}
$$

Table II. - Knight shifts, $T_{1}$ and $T_{1 \rho}$ values of the different lines of figure $2 c$.

\begin{tabular}{|c|c|c|c|c|c|}
\hline $\begin{array}{c}\text { Position } \\
\text { in TMTSF molecule }\end{array}$ & $\begin{array}{c}\text { Knight shift } \\
(\mathrm{ppm})\end{array}$ & $\begin{array}{c}T_{1} \\
(\mathrm{~ms})\end{array}$ & $\begin{array}{c}T_{1 \rho} \\
(\mathrm{ms})\end{array}$ & $\begin{array}{c}K^{2} T_{1} T \\
\left(10^{-6} \mathrm{~s} \mathrm{~K}\right)\end{array}$ & $\eta$ \\
\hline $4,5,14,15$ & -25.2 & 3000 & 8 & - & - \\
& -22.2 & 2800 & 9 & - & - \\
& -10.0 & 5000 & 12 & - & - \\
\hline 6.3 & 4000 & 7 & - & 9.045 & 92.5 \\
& 17 & 530 & 52 & 50.5 \\
$(1,2,11,12$ & 34 & 545 & 50 & 0.071 & 35.5 \\
& 38 & 260 & 37 & 0.117 & 37.8 \\
\hline 3,13 & 137 & 82 & - & 0.454 & 9.15 \\
& 207 & 22 & - & 0.278 & 14.9 \\
\hline
\end{tabular}


where $\chi_{\mathrm{P}}$ is the Pauli susceptibility [7] of the salt, $A_{j}$ is the isotropic part of the hyperfine tensor for the considered carbon (given as about $72 \mathrm{MHz}$ in Ref. [2]), and $\rho_{j}$ the spin density on the site $j$.

The data of relaxation times, $T_{1}$, corresponding to $\mathrm{C}$-sites are also listed in table II. We notice a very large distribution of $T_{1}$ values. In particular the centre carbons come to equilibrium with the lattice in a very short time scale. However these centre carbons cannot influence the relaxation of the corner carbons through cross-relaxation effects because of the strong dilution of naturally abundant ${ }^{13} \mathrm{C}$ spins in the molecule.

Spin densities of TMTSF $^{+0.5}$ have been calculated by Metzger [8] for the related system (TMTSF) ${ }_{2} \mathrm{PF}_{6}$, taking the full symmetry of the crystal into account. Furthermore, spin densities have also been reported for the $\mathrm{TSF}^{+}$cation radical [9]. Both calculations agree in as much as the corner carbons carry small spin densities. Metzger [8] found a distribution in spin densities as confirmed experimentally in this work. The centre carbons $\left(\rho_{\mathrm{C}(3)}=0.0635\right.$ and $\rho_{\mathrm{C}(13)}=0.0666$ [8]) carry larger spin densities in accordance with the larger Knight-shifts, table II. Finally, vanishing spin densities are found on the methyl carbons, for which small but negative Knightshifts are observed.

Thus, qualitatively (and to some extent quantitatively), we find agreement between spin densities in the conducting crystal and estimated densities for the isolated molecule [9] (the apparent negative spin densities of methyl-carbons are discussed below).

The conduction band in (TMTSF) ${ }_{2} \mathrm{ClO}_{4}$ is in a simple description arising from a $\pi$-type MO. ${ }^{13} \mathrm{C}$ hyperfine splittings have been investigated by EPR in planar hydrocarbon radicals [10], and it was found that they can be described as arising from exchange of $2 p_{z}$ and $2 s$, plus an additional term arising from neighbouring $2 \mathrm{p}_{\mathrm{z}}$ densities inducing negative spin densities through sigmabonds. In the framework of Huckel theory a relation

$$
A_{j}=a_{j} \rho_{j}+\sum_{k} a_{j k} \rho_{k}
$$

(where $k$ labels atoms neighbouring the $j$-carbon atoms) was found with values of 30 to 35 Gauss (84 to $98 \mathrm{MHz}$ ) for $a_{j}$ and about -14 Gauss $\left(-39 \mathrm{MHz}\right.$ ) for $a_{j k}$. Thus, ${ }^{13} \mathrm{C} \mathrm{Knight-shifts} \mathrm{are}$ expected to be very sensitive to local surroundings. We can rationalize the negative Knight-shifts on the methyl groups within this model because, (according to Metzger [8]) they carry little $\pi$-density, and the second term in the above relation dominates. Thus the coupling to the corner carbons induces negative Knight-shifts ( $a_{j k}$ in Eq. (2) is negative).

Even though more sophisticated calculations are anxiously awaited, the interpretation of the Knight trunic properties in the conducting solid appear suprisingly « local ». These results are, however, in accordance with a narrow bandwidth [11] (weak intra stack interactions) where the « local » spin densities taken as $\sum_{E_{F}} c_{j}^{2}$, where $c_{j}$ is the coefficient of the $j$-orbital, are not strongly modified in the solid state as compared to the isolated molecule.

This « local » aspect of the electron spin dynamics will be emphasized again in the following section.

\section{The Korringa product.}

We now come to the interpretation of $T_{1}$ values reported in table II. ${ }^{13} \mathrm{C}$-atoms belonging to the methyl groups exhibit remarkably long spin-lattice relaxation times. This is likely to be related to the smallness of the spin density on those carbon atoms. Because the (negative) contribution to the Knight-shift coming from the polarization of the sigma-bonds is dominant for the methyl groups the discussion of their Korringa product, namely the magnitude of $K^{2} T_{1} T$ will not be undertaken. Instead, we focus the discussion on the C-sites exhibiting positive Knight-shifts (i.e. corner and centre carbons). 
Following table II we notice that Knight-shifts and $1 / T_{1}$ values display the general features of nuclear relaxation in electronic conductors [11] : the larger the Knight-shift the faster the relaxation.

However the examination of $K^{2} T_{1} T$ equal to $\left(\frac{\gamma_{\mathrm{e}}}{\gamma_{\mathrm{n}}}\right)^{2} \frac{\hbar}{4 \pi k_{\mathrm{B}}}$ in the simplest picture of a free electron gas reveals an interesting behaviour. First, there is no unique value of $K^{2} T_{1} T$ among all carbons of the molecule. Secondly, $K^{2} T_{1} T$ is very much smaller than the value derived from the Korringa relation. Values of the enhancement factor

$$
\eta=\left(\frac{\gamma_{\mathrm{e}}}{\gamma_{\mathrm{n}}}\right)^{2} \frac{\hbar}{4 \pi k_{\mathrm{B}}} / K^{2} T_{1} T
$$

are listed in table II.

There exists a broad distribution of $\eta$ in the molecule, but crudely speaking the values belong to two groups amounting to $\approx 12$ and ranging from 37 up to 92 for centre and corner carbons, respectively.

The enhancement of the nuclear spin relaxation mechanism in 1-D conductors is a well known feature which has been related to the fact that $1 / T_{1}$ is essentially determined by the value of the component of the electron susceptibility $\chi(\mathbf{q})$ at $q \approx 2 k_{\mathrm{F}}$ [13]. Hence, $\left(T_{1} T\right)^{-1}$ is usually more enhanced than the Knight-shift which derives from the uniform susceptibility $\chi(q=0)$.

We may notice that for two corner atoms out of the four the enhancement factor $(\approx 46)$ is about similar to the enhancement factor measured for the corner atoms of the parent compound TTFTCNQ [14]. In this compound the $1 / T_{1}$ enhancement has been attributed to Coulomb repulsive energy being of the order of the longitudinal bandwitdh. Inspection of table II shows that the relaxation rate is " more enhanced " for the corner than for the centre carbons.

We feel unable at present to provide a satisfying interpretation for such an observation. However we suggest as a possible starting point the following simple picture.

The molecular wave function giving rise to the conduction band exhibits nodes both between $\mathrm{C}(2)-\mathrm{Se}$ and Se-C(3) atoms in the molecule [15]. This means that the starting molecular orbitals are essentially decoupled and that it may be equally valid to build the bands (or subbands) along the stacking direction from the intermolecular overlap of respective atomic orbitals. In such a situation the subbands related to the corner carbons would be much narrower (the intermolecular overlap is smaller) than the subbands coming from the centre carbons. As correlation effects play a larger role in narrower bands, this may explain the difference between Korringa products oi i $n n:=$ and centre carbons. The previous argument can be pushed one step further and serve to discriminate between corner carbons which are close or far from $\mathrm{ClO}_{4}^{-}$anions. $\mathrm{C}$-atoms close to $\mathrm{ClO}_{4}^{-}$ groups (A atoms in Table II) are expected to present both larger Knight-shifts and $1 / T_{1}$ because of the larger electron density.

\section{Conclusion.}

To summarize, the ${ }^{13} \mathrm{C}$ high resolution NMR investigation of (TMTSF) ${ }_{2} \mathrm{ClO}_{4}$ has enabled us to resolve all individual C-sites of the organic molecule in the (TMTSF) ${ }_{2} \mathrm{ClO}_{4}$ crystal. The distribution of Knight-shifts shows that the spin density is inhomogeneous, in qualitative agreement with the calculations by Metzger [8]. However, the simple calculation cannot account directly for the observed Knight-shift distribution. Consequently, more elaborate computations of spin and charge distributions in the (TMTSF) ${ }_{2} \mathrm{X}$ series should be undertaken.

We have also suggested that the deviation of $1 / T_{1}$ from the Korringa behaviour supports a picture in which Coulomb correlations are dominant for corner atoms as compared to the atoms in the central double bond. Finally, the Letter has presented the first ${ }^{13} \mathrm{C}$ high resolution NMR 
study of a conductor of the (TMTSF $)_{2} \mathrm{X}$ series. It is the preliminary report of a more extensive high resolution NMR program which is being carried on with (TMTSF) ${ }_{2} \mathrm{X}$ systems.

\section{Acknowledgments.}

One of us (RJS) wishes to thank the CNRS for financial support and GDPC, Montpellier for its hospitality.

\section{References}

[1] Bechgaard, K., Carneiro, K., Rasmussen, F. B., Olsen, M., Rindorff, G., Jacobsen, C. S., PederSEN, H. J. and SCOTt, J. C., J. Am. Chem. Soc. 103 (1981) 1140.

[2] Mehring, M. and Spengler, J., Phys. Rev. Lett. 53 (1984) 2441.

[3] Bechgaard, K., Cowan, D. O. and Bloch, A. N., J. Chem. Soc. Chem. Commun. (1974) 937.

[4] Mehring, M., Principles of High Resolution NMR in Solids (Springer-Verlag, New-York) 1983.

[5] Kistenmacher, T. J., Emge, T. J., Shu, P., Cowan, D. O., Acta Crystallogr. Sect. B 35 (1979) 772.

[6] Rindorff, G., Soling, H., Thorup, N., Acta. Crystallogr., Sect. B 38 (1982) 2805.

[7] Miljak, M., Cooper, J. R. and BechgaARd, K., report $1.4 * 10^{-4}$ emu/mole in J. Physique Colloq. 44 (1983) C3-893.

[8] Metzger, R. M., J. Chem. Phys. 75 (1981) 482.

[9] Kinoshita, N., Tokumoto, M., Anzai, H., Ishiguro, T., Saito, G., Yamabe, T. and Terame, T., J. Phys. Soc. Jpn 53 (1984) 1504.

[10] Carrington, A. D. and Mclachlan, A. D., Introduction to Magnetic Resonance (Harper \& Row and John Weatherhill) 1967, p. 93.

[11] Jacobsen, C. S., Tanner, D. B. and BechgaArd, K., Phys. Rev. Lett. 46 (1981) 1142.

[12] Slichter, C. P., Principles of Magnetic Resonance (Springer-Verlag, New-York) 1980.

[13] Soda, G., Jerome, D., Weger, M., Alizon, J., Gallice, J., Robert, H., Fabre, J. M. and Giral, L., J. Physique 38 (1977) 931.

[14] Takahashi, T., Jerome, D., Masin, F., Fabre, J. M. and Giral, L., J. Phys. C 17 (1984) 3777.

[15] Berlinsky, A. J., Carolan, J. F. and Weiler, L., Solid State Commun. 15 (1974) 795, and reference [8]. 